\title{
Evaluation of the Prevalence of Human Papillomavirus in Asymptomatic Patients at the Women's Clinic in Hamadan and Comparing the 2 Methods of Pap Smear and PCR in Detecting the Virus
}

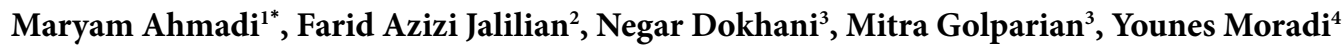

\begin{abstract}
Objectives: Human papillomaviruses (HPVs) are a group of non-enveloped viruses which contain double-stranded DNA, and have widespread prevalence in human populations. The aim of this study was to evaluate the prevalence of HPV in asymptomatic patients at the Women's Clinic in Hamadan and compare the two techniques of Pap smear and Polymerase chain reaction (PCR) in detecting the virus.

Materials and Methods: This epidemiologic study was carried out on 1770 asymptomatic patients at the Women's Clinic in Hamadan. After collecting the cell samples from the cervical areas, they were placed in a pre-prepared medium by a physician and divided into two sections after being sent to the laboratory; the first part was for Pap smear and the second for PCR. If there was a viral genome, it could be determined by PCR. People who were HPV + were candidates for typing.

Results: The results of the present study showed that out of 1770 patients aged between 14 and 60 years, referred to Hamadan health centers, 271 patients (15.31\%) had HPV. Among them, the highest number of HPV+ cases belonged to the age group of 31-40 years, with the lowest number belonging to the age group of 15-20 years old. Furthermore, out of $271 \mathrm{HPV}+$ patients, 111 (40.09\%) had abnormal Pap smear and more than 90\% had HPV infection, while less than $10 \%$ had low risk (LR) HPV.

Conclusions: The results of this study showed that the prevalence of HPV in Hamadan health centers was relatively high. According to the results of this study, it can be concluded that the use of PCR along with Pap smear can help the patients with diagnosis and better treatment.

Keywords: HPV, Prevalence, Pap smear, PCR, Asymptomatic patient
\end{abstract}

\section{Introduction}

Human papillomaviruses (HPVs) are a group of nonenveloped viruses, with a small double-stranded DNA and widespread prevalence in human populations (1). The virus infects squamous epithelial cells of the skin, upper respiratory tract mucosa, and anogenital area (2). Cervical cancer is the fourth most common cancer among women and the second most common cancer among women between the ages of 14-44. According to statistics, in developing and developed countries, the prevalence of HPV infection is $44.2 \%$ and $22.6 \%$, respectively (3). Low age at the time of the first sexual intercourse (less than 16 years of age), having multiple sexual partners, smoking, race, high parity, low socioeconomic status, and chronic suppression of the immune system are among the most important risk factors that strongly contribute to cervical cancer (4). Vaginal bleeding is the most common symptom seen in patients with cervical cancer. In most cases, postcoital bleeding arises after any intercourse, which may be observed irregularly or after menopause (4). In over $90 \%$ of cervical cancers, persistent infection is observed with high risk types of HPV (4). For the malignancy of a normal cell, an expression of the E6 and E7 antico-proteins interacting with the repressive genes of the tumor, P53 and $\mathrm{Rb}$, is required (5). People who are infected with low risk HPV (LR-HPV) usually have no symptoms $(1,4)$.

One of the most cost-effective methods for diagnosis of cervical cancer is the Pap smear test. Pap smear screening tests are done to detect pre-cancerous and cancerous changes in the endocervical canal of the uterus, in which unusual squamous cells or neoplastic lesions in the cervix are detected $(6,7)$. In healthy and symptom-free women, the Pap smear test is the first diagnostic step, but in every Pap smear test, there is a $50 \%$ chance of error, in a false negative way; therefore, the Pop smear negative outcome can never be relied on (7). Considering these, an accurate method for identifying types of HPV could 
be the use of polymerase chain reaction (PCR), in which not only the previous types but also new types of the virus are detected. Regarding the Pap smear test and PCR test, in some studies, some people with negative Pap smears had a variety of HPVs in their cervix as detected by a simultaneous PCR technique $(8,9)$.

A number of DNA viruses have been isolated from different parts of the body of healthy people. HPV that is isolated from healthy vagina is one of these DNA viruses (5). In this study, we investigated the prevalence of HPV in healthy people referred to health centers of Hamadan and compared the two methods of Pap smear and PCR, as well as investigating the HPV virus as a normal viral flora.

\section{Materials and Methods}

Subjects

The present epidemiologic study was conducted on 1770 asymptomatic patients in Hamadan hospitals who had referred for performing Pap smear. The married women who had an annual check-up and had no underlying illness, pain, and abnormal vaginal bleeding were included in this study, while those with a history of genital diseases or any treated genital disease were excluded.

\section{Study Method}

After collecting the cell samples from the cervical areas, they were placed in a pre-prepared plate by a physician and divided into two sections after being sent to the laboratory; the first part was for Pap smear and the second part for PCR.

\section{Method of Performing Pap Smear}

Pap smear was done in two forms; a sample in a liquid medium and a lamina, each of which is about $30 \%$ probable. Pap smear began with preparing and staining the cells. Then the cells were examined by a pathologist, and the following forms could be observed: (a) healthy or $(b)$ unspecified atypical squamous cells, and (c) lowgrade squamous intraepithelial lesion (LSIL). High-grade squamous intraepithelial lesions (HSILs) and squamous cell carcinoma, which if koliocytes are seen, are signs of neoplasia, which is characterized by an increase in the size of the nucleus to 2-3 times the normal size, the appearance of a darkened nucleus, and a bright area which is near the core of the perinuclear halo.

The second part of the sample, which was isolated for PCR, was centrifuged and then underwent the extraction procedure. We used a high-pure viral nucleic acid kit (Roche Company) to extract the genetic material. After extraction in terms of sample concentration, we examined if there was a sufficient concentration of the genome. Then we used NanoDrop to remove $1 \mu \mathrm{L}$ of the sample and determine the concentration and purity. If there was a viral genome, it could be determined by PCR. Women who were $\mathrm{HPV}^{+}$were candidates for typing (Table 1 ).
Table 1. HPVL1 and $\beta$-actin Primer Sequences

\begin{tabular}{ll}
\hline Genes & Primer Sequence $\left(\mathbf{5}^{\prime} \rightarrow \mathbf{3}^{\prime}\right)$ \\
\hline \multirow{2}{*}{ HPVL1 } & Forward: GCM CAG GGW CAT AAY AAT GG \\
& Reverse: CGT CCM AAR GGA WAC TGA TC \\
\multirow{2}{*}{ B-actin } & Forward: GAA GAG CCA AGG ACA GGT AC \\
& Reverse: CAA CTT CAT CCA CGT TCA CC \\
\hline
\end{tabular}

Sample Calculation Method

According to previous studies, if the prevalence of HPV was $40 \%$, with a precision of $3 \%$ and a $99 \%$ confidence level for 1770 women, this study could be conducted.

$N=\frac{Z^{2} 1-\alpha P(1-P)}{d^{2}}$

Data Analysis

Demographic factors such as sex, age, education are shown as percentage. Given as the cross-sectional study, logistic regression was used to examine the relationships between other variables and positive or negative results of the experiment.

\section{Results}

The results of this study showed that out of 1770 women referred to Hamadan health centers, 271 patients (15.31\%) were $\mathrm{HPV}+$, from which the highest number of $\mathrm{HPV}+$ cases belonged to the age group of 31-40 years, while the lowest number belonged to 15-20 years old.

Moreover, regarding the level of education, the results showed that women with a lower level of education (less than high school) belonged to the group with the highest number HPV+ cases, while those who had higher education level (college educated) belonged to the group with the lowest number of HPV+ cases (Table 2).

The study of marital status among the patients showed that the highest number of women who had never been married were those who rarely lived with their parents.

Our results also showed that most people with HPV had no birth control during sexual intercourse.

Moreover, our study on contraceptive methods in people with HPV showed that condom use was a more reliable method than other methods, including OCP and IUD.

The prevalence and type of HPV were assessed by both methods of Pap smear and PCR in women who were regularly checked in Hamadan health centers. Out of $271 \mathrm{HPV}+$ women aged between 14-60 years old, 111 (40.09\%) had abnormal Pap smear. More than 90\% were detected with high-risk (HR) HPV infection, while less than $10 \%$ were detected with LR-HPV infection (Table 3).

\section{Discussion}

The results of this study showed that $15.31 \%$ of the patients referred to the Hamadan health centers had HPV. Among them, the highest number of cases belonged to 


\begin{tabular}{lll}
\multicolumn{2}{l}{ Table 2. Demographic Information of Participants in the Study (N=1770) } \\
\hline & Sample Size & $\begin{array}{l}\text { Prevalence, } \% \\
\text { (95\% Cl) }\end{array}$ \\
\hline Age & 271 & $15.31 \%$ \\
HPV+ cases & 4 & $1.47 \%$ \\
$15-20$ & 17 & $6.27 \%$ \\
$21-25$ & 58 & $21.40 \%$ \\
$26-30$ & 95 & $35.05 \%$ \\
$31-40$ & 57 & $21.03 \%$ \\
$41-50$ & 40 & $14.76 \%$ \\
$51-60$ & & \\
Marital status & 40 & $14.76 \%$ \\
Married & 81 & $29.88 \%$ \\
Divorced, separated & 115 & $42.43 \%$ \\
Never married & 35 & $12.91 \%$ \\
Living with partner & & \\
Education level & 124 & $45.75 \%$ \\
< High school & 82 & $30.25 \%$ \\
High school and GED & $82.98 \%$ \\
> High school & 65 &
\end{tabular}

the age group of 31-40 years and the lowest number were between the ages of 15-20 years. Dunne et al found that the prevalence of HPV among 1921 American women was $26.8 \%$ based on PCR results. Considering their ages, $24.5 \%$ were between the ages of $14-19,44.8 \%$ were aged 20-24 years, $27.4 \%$ were aged $25-29$ years, $27.5 \%$ were between 39-39 years, 25\% were 40-49 years old, and $19.6 \%$ of infected people were in the age range of 50-59 years. The rate of infection was decreased in vaccinated types (types $6,11,16,18$ ). However, due to the increase in sexual partnerships and sexual activity in the ages of 20-24, the infection rate was higher than the estimated level (10). Additionally, Bernabe-Dones et al in the United States in 2016 reviewed the issue of high incidence of HPV in colorectal cancer in Spaniards. From 81 subjects with a mean age of 60.9 years, 45 were with colorectal cancer and 36 were the control patients. High number of the patients (44.2\%) with colorectal cancer were infected with HPV and only one out of 36 controls (2.8\%) was positive

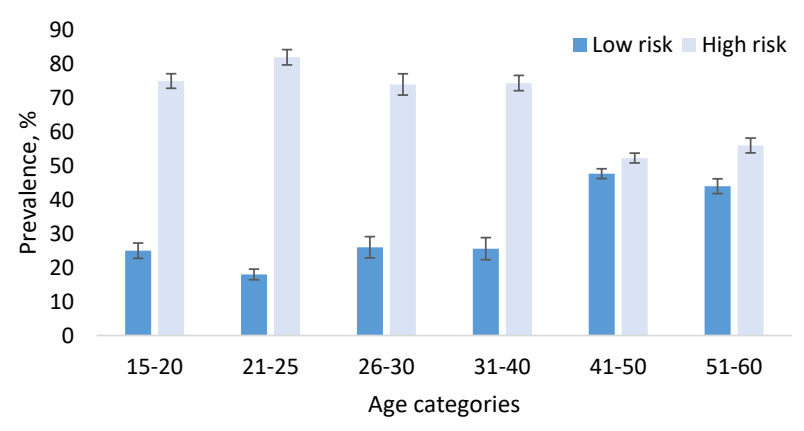

Figure 1. Prevalence of Low-Risk and High-Risk HPV Types Among Females Aged 15 to 60 Years.

for HPV (11). Considering the marital status among the patients, it was observed that the highest number of never-married women rarely lived with their parents. In addition, in terms of education level, the results showed that the highest number of women with HPV had a lower level of education (less than high school), while college educated women belonged to the group with the lowest number of HPV+ cases. This conclusion reveals the impact of education level and awareness, therefore the higher the level of awareness of the women, the lower the risk of HPV. A study by Dunne et al displayed that the incidence of HPV among individuals with low literacy rates was higher than that among those with a high level of education. The HPV incidence was also high among those who were never married compared to the other participants in the study (10).

Based on other studies, most people who had HPV had used no contraceptive method during sexual intercourse. Our study on contraceptive methods in people with HPV showed that condom use was a more reliable method than other methods, including OCP and IUD. The study of Dursun et al in Turkey in 2009 demonstrated that the prevalence of HPV in 403 Turkish women was high (23\% $\mathrm{HPV+}$ (93 participants with an abnormal cytology and 310 with a normal cytology). Moreover, $36 \%$ of people with abnormal Pap smear and 20\% of people with normal Pap smear were infected with HPV. The rate of infection

Table 3. Distribution of HPV Type Based on Cytological Results

\begin{tabular}{|c|c|c|c|c|c|c|}
\hline Infection Group & Genotype Risk & WNL & ASCUS & LSIL & HSIL & Total \\
\hline HPV positive & & $154(56.82 \%)$ & $52(19.18 \%)$ & 45 (16.6\%) & $20(7.3 \%)$ & 271 \\
\hline HPV31, 33, 35, 39, 45, 51, 52, 56, 58, 59, 66, 68 & $\mathrm{HR}$ & $110(61.79)$ & $32(17.9 \%)$ & $22(12.35 \%)$ & $14(7.8 \%)$ & 178 \\
\hline HPV6, 11, 42, 43, 44 & LR & $71(62.8 \%)$ & $20(17.6 \%)$ & $18(15.2 \%)$ & $4(3.5 \%)$ & 113 \\
\hline HPV16 & $\mathrm{HR}$ & $30(44.1 \%)$ & $16(23.5 \%)$ & $17(25 \%)$ & $5(7.3 \%)$ & 68 \\
\hline HPV18 & $\mathrm{HR}$ & $15(50 \%)$ & $10(33.3 \%)$ & $5(16.6 \%)$ & $0(0.0 \%)$ & 30 \\
\hline HR HPV positive & HR & $120(54 \%)$ & $48(21.6 \%)$ & $40(18.01 \%)$ & $14(6.3 \%)$ & 222 \\
\hline LR HPV positive & LR & 47 (85.45\%) & 4 (7.2\%) & $4(7.2 \%)$ & $0(0.0 \%)$ & 55 \\
\hline
\end{tabular}

WNL: Within normal limits, ASCUS: atypical squamous cell, LSIL: Low-grade squamous intraepithelial lesion, HSIL: High-grade squamous intraepithelial lesion, HR HPV: High risk human papilloma virus, LR HPV: Low risk human papilloma virus. 
of normal women was as follows: HPV 16 (36\%), HPV $6(22 \%)$, and HPV 18 (13\%). In women with abnormal Pap smear, the infection rate was HPV 16 (35\%), HPV 6 (19\%), and HPV 18 (8\%). In general, the infection rate for HPV was higher than the estimated level (8).

In 2012 Eide et al found that positive genotype of HPV can obviously improve management of patients after pathologic cytology.

\section{Conclusions}

The results of this study showed that the prevalence of HPV in Hamadan health centers was relatively high. According to the present study, it can be concluded that the use of PCR in addition to Pap smear can help the patients with HPV diagnosis and better treatment.

\section{Conflict of Interests}

Authors declare that they have no conflict of interests.

\section{Ethical Issues}

This study was approved by the ethical committee of Hamadan University of Medical Sciences.

\section{Financial Support}

This study was supported by the Research and Technology Deputy of Hamadan University of Medical Sciences.

\section{References}

1. Ma Y, Madupu R, Karaoz U, et al. Human papillomavirus community in healthy persons, defined by metagenomics analysis of human microbiome project shotgun sequencing data sets. J Virol. 2014;88(9):4786-4797. doi:10.1128/ jvi.00093-14

2. Mammas IN, Sourvinos G, Zaravinos A, Spandidos DA. Vaccination against human papilloma virus (HPV): epidemiological evidence of HPV in non-genital cancers.
Pathol Oncol Res. 2011;17(1):103-119. doi:10.1007/s12253010-9288-0

3. Malary M, Moosazadeh M, Hamzehgardeshi Z, Afshari M, Moghaddasifar I, Afsharimoghaddam A. The prevalence of cervical human papillomavirus infection and the most atrisk genotypes among Iranian healthy women: a systematic review and meta-analysis. Int J Prev Med. 2016;7:70. doi:10.4103/2008-7802.181756

4. Jonatan S, Berek J. Novak's Gynecology. 14th ed. New York: Lippincott Williams and Wilkins Publishers; 2012.

5. Yap T, Watkin N, Minhas S. Infections of the genital tract: human papillomavirus-related infections. Eur Urol Suppl. 2017;16(4):149-162. doi:10.1016/j.eursup.2016.08.005

6. Apgar BS, Brotzman G. HPV testing in the evaluation of the minimally abnormal Papanicolaou smear. Am Fam Physician. 1999;59(10):2794-2801.

7. Shader RI. The PAP test and the pap smear. Clin Ther. 2015;37(1):1-3. doi:10.1016/j.clinthera.2014.12.002

8. Dursun P, Senger SS, Arslan H, Kuşçu E, Ayhan A. Human papillomavirus (HPV) prevalence and types among Turkish women at a gynecology outpatient unit. BMC Infect Dis. 2009;9:191. doi:10.1186/1471-2334-9-191

9. Ghaffari SR, Sabokbar T, Mollahajian H, et al. Prevalence of human papillomavirus genotypes in women with normal and abnormal cervical cytology in Iran. Asian Pac J Cancer Prev. 2006;7(4):529-532.

10. Dunne EF, Unger ER, Sternberg M, et al. Prevalence of HPV infection among females in the United States. JAMA. 2007;297(8):813-819. doi:10.1001/jama.297.8.813

11. Bernabe-Dones RD, Gonzalez-Pons M, Villar-Prados A, et al. High prevalence of human papillomavirus in colorectal cancer in Hispanics: a case-control study. Gastroenterol Res Pract. 2016;2016:7896716. doi:10.1155/2016/7896716

12. Eide ML, Debaque H. HPV detection methods and genotyping techniques in screening for cervical cancer. Ann Pathol. 2012;32(6):e15-e23. doi:10.1016/j. annpat.2012.09.231

(c) 2020 The Author(s); This is an open-access article distributed under the terms of the Creative Commons Attribution License (http:// creativecommons.org/licenses/by/4.0), which permits unrestricted use, distribution, and reproduction in any medium, provided the original work is properly cited. 